\title{
Leprosy and "A Disease Called Leprosy"**
}

\author{
T. A. STRINGER \\ United Kingdom Activities Officer, LEPRA, London W.1.
}

\begin{abstract}
Despite arguments put forward in Leprosy Review (1972) 43, 69-105, there is a case for retaining the substance of current terminology related to leprosy, particularly because of its value to fund-raising. In any event, the public will not co-operate in the adoption of new terminology, but find a vital rôle for the linguistic associations of leprosy. Acknowledging the nature of general knowledge about the "disease called leprosy", we have opportunities of educating the public in both endemic and non-endemic countries about the true nature of the disease. Identifying the nature of semantic confusion involved in the Christian motivation towards leprosy sufferers, we may be able to influence the public reaction to leprosy by correcting the old terminology in religious texts, and by referring to the defined disease as "actual leprosy".
\end{abstract}

In Leprosy Review (1972) 43, 69-105, various aspects of leprosy are examined in a range of articles from the superficial to the scientific. Taken as a whole, these articles reveal extraordinary conflicts within the world of leprosy workers and suggest, between the lines, that a continuous close proximity to the disease may not be conducive to a factual assessment of its rôle in the public sphere.

We are dealing with a disease which occurs in a number of countries, of different cultures, traditions and languages. Moreover, we have to take into account that the traditions associated with leprosy carry the local history into the present in each country. In such circumstances, generalizations are foolhardy, and subjective conclusions dangerous, particularly if they invite world-wide participation in costly exercises of verbal manipulation which are not by any means guaranteed to solve local problems and which would create problems elsewhere.

It is perhaps a little to one side of the purpose of Leprosy Review to suggest that those engaged on raising funds for anti-leprosy work may have a case to present in this area of controversy as to public reaction to the disease, but since it is acknowledged that voluntary agencies have a rôle. in field work, it seems only fair to consider some aspects of the methods which enable them to fulfil this rôle, and indeed, some of the problems which they face, in arousing public attention for a forgotten, unfashionable and undramatic cause.

Fund-raising for leprosy in the United Kingdom is competitive, with some 77,000 charities vying for a share of public goodwill and generosity. The vast majority of these are small, local efforts, to serve a local purpose. Appeals for world-scale humanitarian purposes invite an instinctive resistance, justified by the authority of the quotable, if questionable, aphorism "Charity begins at home". To go beyond this barrier, an "overseas" charity must involve the potential donor

* Received for publication January, 1973. 
in thought and in feelings of obligation which pre-suppose some philosophical standpoint, however undefined. The clarity of appeals is their greatest chance of success, and since the public is ill-informed about leprosy, nonetheless adhering to traditional beliefs about a "disease called leprosy", details of which have never been assimilated, the evocation of a reaction to the word "leprosy" is an essential factor in stating the case. "Leprosy" is related to Biblical and mediaeval traditions, and is thought by many to have "died out" centuries ago. Appeals must, therefore, correct these misunderstandings before they can hope to present true current information. The process is one of relying on the existence of misunderstanding, and making the correction reveal the case for concern and generosity. It is a process of associating the traditional "discase called leprosy" with the actual currently defined disease to provide for the dissociation of fiction from fact. To attempt to do this without a total reliance on the abundant misuse of the word "leprosy" would be a complex and vain exercise and would, moreover, lose the interest and imagination of the public, who are saturated with information, most of which is irrelevant to any of their needs or interests. Because of all the factors which frustrate the efforts of field workers, the word "leprosy" invites curiosity and attention, and provides for a strategy in gaining support. This seems a legitimate and harmless process. In a similar way, pictorial representation of leprosy sufferers provides immediate information about the nature of the disease, but, stressing the unaesthetic aspects of it, seems calculated to reinforce its stigma.

Fund-raisers are, then, faced with a considerable dilemma. Do their efforts to provide funds for treatment and research perform a disservice to their medical colleagues, prolonging traditions, the redundancy of which is eagerly awaited in the field? It is a dilemma which cannot be resolved if the answer is affirmative, for a loss of income must affect the anti-leprosy effort adversely. And so it is legitimate to refer the quandary back to those who use the funds and ask if they would be prepared to do without such financial assistance as can be offered in favour of obtaining a new identity for the disease?

We take it for granted that the more money we can provide, the greater will be the momentum of treatment and research programmes, and from this increased momentum, eventual conquest will result. Even against the background of implicit disapproval, we have to continue to rely on the dynamic of an appeal for participation in a world-wide campaign to overcome an ancient enemy, which, when understood, is far less fearsome than its legends suggest. The pursuit of these endeavours is conducted on the basis of evidence of public misunderstanding, and of the need and opportunity to correct it, arising out of just the kind of hysterical clumsiness embodied in the Brazilian headlines referred to (Rotberg, 1972). "Hippies espalham a lepra" has its United Kingdom equivalent "Immigrants bring in leprosy", but such exaggerations invite correction from authoritative bodies, and serve to further the education of the reading public. Journalistic irresponsibility is very much here to stay all over the world and may be relied upon to perpetuate the damaging myths of leprosy, as well as other diseases and conditions. Rather than shrug our shoulders at the profundity of malevolence or ignorance of this kind, we take advantage of the occasion to put a factual case to a public whose interest has, however sensationally, been aroused. Such opportunities are infrequent, and it is certainly more difficult to get correct information printed for its own sake, since there exists a paradoxical conviction among newspaper editors which denies that there is any public interest in the disease. 
Such a stance as that of journalists assumes the existence of some degree of public stigma of leprosy, and it is in this connection that the paper of Gussow and Tracy (1972) is of particular interest. Granted that all circumstances in the United States do not apply elsewhere, the fact that the presumption of stigma by anti-leprosy workers is not borne out by scientific investigations suggests the need for the provision of as much evidence about other countries before conclusions can be reached, and certainly before educative programmes are drawn up. The paper is an invitation to us all to reassess our assumptions of positive public attitudes, and a warning that we may be wide of the mark in the particular area of assuming universal hostility towards leprosy and the leprosy sufferer. The suspicion arises that it may be our own familiarity with leprosy and its traditional associations which makes us ascribe traditional reactions to the public which is, in fact, indifferent, and which does not replace out-dated reactions with any attitude towards actual leprosy, so much as maintains a familiarity with a "disease called leprosy," which is of curiosity and historical in terest. The strength of appeals based on this assumption may begin to emerge, and equally the opportunity of supplanting myth with fact, emphasizing that the exaggeration in the myth has no application in consideration of the actual disease. So, we may be able to achieve a possible objective, of defining actual leprosy as a "mildly contagious condition, effectively and cheaply checked by prompt and regular treatment" only because, in doing so, we can deny its high contagiousness and incurability, both of which are assumed by the public.

These considerations of the strategy applied to fund-raising in non-endemic countries may seem irrelevant to the problems faced by anti-leprosy workers in endemic countries. But certain aspects of the situation appear to be universal, not least among these the lack of tangible evidence of the degree or nature of stigma. Even accepting that the stigma was intensely felt in all endemic countries, should not the same strategy apply as that which seems to work among indifferent and unthreatened people elsewhere?

The populations of endemic countries divide themselves into the suffering and the non-suffering, and the latter group has two definitions. They are at once potential victims and potential repositories of attitudes towards actual victims. Resistance to educative measures which aim at protecting them from a hazard has a number of counterparts in many societies. The dangers of drug-taking, smoking, alcoholism and road accidents all seem to fall into a category of items about which the public would rather not think at all, but the significant difference between leprosy and these other hazards is that the resistance to knowledge is based in this case on false assumptions rather than on facts chosen to be ignored. Programmes of propaganda based on the slogan "Leprosy is NOT ..." therefore have an immediate advantage over measures in other areas which have to create information from the outset. The same applies to dealing with non-suffering endemic populations in respect of their attitude towards leprosy sufferers. Again, the essential ingredient of such campaigns is the acknowledgment of existing public misunderstanding and the conception of this as an opportunity for correction, and this necessitates an identification of the disease which is misunderstood, by the name by which it is misunderstood.

Inevitably, this leads us to further consideration of the absorbing paper of Rotberg (1972) and to the debate on nomenclature. One approaches such a paper with profound humility, and with acknowledgment that the deeply felt plea is for help with a local problem. This in turn invites a desire to agree with the 
recommendations, but, despite the wide catalogue of arguments rebutted, the paper omits a vital consideration. What happens to the terminology of leprosy after the successful adoption of a substitute vocabulary?

We have to accept that traditional misunderstandings about leprosy are embodied in the cultures of both currently endemic and non-endemic countries. These misunderstandings have given connotations to a range of terms whose medical usage is lost on the public at large, but which form valuable instruments of communication, particularly in the descriptive area (Skinsnes and Elvove, 1970). Such usages will not die out, since they are based on casually acquired misinformation and the correction of terminology in restricted circles will not cause so much as a ripple on the mainstream of public usage. This is evidenced by the headline which includes the word "leprosos" despite efforts, which we realize have been considerable, for the exclusion of the word, and in the United Kingdom, even among informed supporters of anti-leprosy work, as well as among politicians and footballers, the word "leper" perhaps only used for its conciseness, and dead though it may be in medical circles, will not conveniently lie down in public. It is perhaps worth saying in parentheses that the suggested portmanteau "I Ianseniasis-formerly leprosy" just as any suggested alternative single word, suffers in a comparison with the methods used in currency changes because of the lack of opportunity for the public at large to exercise with the new term, which is vital to any process of linguistic change.

Accepting that we are few indeed to attempt to take on the entire world population in this question of linguistic misuse, might we not do well to consider some of the factors in the creation of the problem to see if there may be other influences which might have a more profound effect in obviating the need for public re-education as to the true nature of leprosy?

The paper from Brazil already cited listed a number of influences which persist in propounding the legends of leprosy as opposed to its actual nature and referred among these to religious instruction. It is surely here that all the problems begin and to this same point that they all return. Certainly the Christian motivation in anti-leprosy work has effectively covered the endemic world, so that even cultures where other traditions are in play have been made aware of the special significance given to succouring leprosy victims by the Christian Church.

It is agreed that leprosy as referred to in the Bible is nowhere necessarily-and in some places certainly not-leprosy as currently clinically defined (Browne, 1970). The attention that Christianity has paid to Biblically defined "lepers" has reflected the vagueness of the original terminology over the ages. It is only after 1873 , at a time when it became possible to define leprosy by reference to its presumed causative agent, that it became "the disease caused by Mycobacterium leprae", and Christian, or any other motivation, could be related to this specific disease. That the disease did not change because of the definition is self-evident, as it is that the disease was the subject of investigation and concern beforehand, but the particular concern for "lepers" reached a crossroads at a point in time when "lepers" became only those who suffered from the specific defined disease. That these people were at best a portion of those for whom the original concern was urged is also agreed (Browne, 1970). It is the most extraordinary and paradoxical coincidence that one modern manifestation of concern originates in 1874 with the foundation of The Leprosy Mission, whose contribution towards the anti-leprosy campaign has been properly acclaimed.

Once leprosy sufferers could be defined in the modern sense, the nature of the 
Christian motivation towards them embodied all the Biblical connotations of the disease called leprosy into their identity, at a-time when, if ever, their identity might have changed. Further, the. evangelization of leprosy sufferers added another contribution to their list of isolating qualifications. It has, through the desire to evangelize and through misconception, been in the interest of Christians to see modern leprosy sufferers as "Biblical lepers", and we have only to consider the rôle that missionary doctors have played and continue to play in effective field work to accept that this interest will continue, and will go on inviting the same confusion as to the similarity between those who suffered from the disease called leprosy and those who are today's leprosy sufferers.

It seems redundant to observe that the terminology of leprosy should have been varied at the time of Hansen's discovery, but such a shift would have emphasized the medical factors in the leprosy sufferers' identity. As it is, a hundred years of reinforcing the semantic confusion has taken us beyond the point where verbal manipulation stands any chance of success, and where it would not deny us the vestigial consciousness of the disease's existence in non-endemic, fund-providing communities. The cart is now truly before the horse, and we might do well to think of ways of isolating the associations of the "disease called leprosy" from those of actual leprosy, as a means of avoiding reliance on public co-operation which will certainly not be forthcoming.

In the first place, it should be possible to impress appropriate religious authorities with the desirability of eschewing reference to leprosy in reproductions of religious texts. This would ensure that future generations will not associate out-worn connotations with the actual disease. Such measures as have already been taken in this direction (Browne, 1970) leave enough to be desired to deny their value altogether. Secondly, reference to "actual leprosy" whenever appropriate in publications of all kinds would effectively dissociate "the disease called leprosy" from the disease as defined. The epithet carries a strong sense of "current" in Romance and non-Romance languages.

If there is a further contribution that anti-leprosy workers in all areas of the subject can accomplish "at a stroke" it is through a determination not to perpetuate leprosy myths by exaggerating the significance of uninformed statements. Incidents of the kind quoted by Pedley (1972) generalize by implication from insignificant episodes, and create myths of public reaction among leprosy workers that outweigh the myths of leprosy among the public.

\section{References}

Browne, S. G. (1970). Leprosy in the Bible.

Gussow, Z. and Tracy, G. S. (1972). The phenomenon of leprosy stigma in the continental United States. Lepr. Rev. 43, 85.

Pedley, J. C. (1972). The stigma of leprosy-in four countries. Lepr. Rev. 43, 94.

Rotberg, A. (1972). The serious Latin American problems caused by the complex "Leprosy, the Word, the Disease" and an appeal for World co-operation. Lepr. Rev. 43, 96.

Skinsnes, O. K. and Elvove, R. M. (1970). Leprosy in society v. leprosy in occidental literature. Int. J. Lepr. 38, 294. 